Vol. 3, No. 1, January 2018, 41-44

Webpage: http://rheumres.org

Email: editor@rheumres.org

\title{
Concurrent psoriasis and gout
}

\author{
Nayyerah Saadati ${ }^{1}$, Bahram Naghibzadeh ${ }^{2}$, Zeinab Saremi ${ }^{{ }^{*}}$ \\ ${ }^{1}$ Ghaem Medical Center, Mashhad University of Medical Sciences, Mashhad, Iran \\ ${ }^{2}$ Rheumatic Diseases Research Center, School of Medicine, Mashhad University of Medical Sciences, Mashhad, Iran
}

\begin{abstract}
Hyperuricemia is reported to have a high prevalence in patients with psoriasis. This metabolic derangement can be associated with the development of gout in such patients. Presented herein is the case of a 53-year-old male who referred to the hospital because of worsening of skin lesions and multiple swollen and painful joints. Physical examination showed symmetric involvement of the patient's knee, first metatarsophalangeal, and subtalar joints. Knee fluid aspiration revealed numerous monosodium urate monohydrate (MSUM) crystals. The patient's serum uric acid level was $10 \mathrm{mg} / \mathrm{dL}$. Kidney function was normal. Although he had experienced a similar episode two years earlier with involvement of the first metatarsophalangeal joint, he did not seek medical attention then and was not receiving prophylaxis for gout. Treatment with prednisone, allopurinol, colchicine, and sulfasalazine resulted in improvement of the symptoms. The patient was followed for six months and no relapse in his skin conditions or gouty attacks were observed. It is prudent to measure uric acid levels in psoriatic patients. Psoriatic patients with hyperuricemia, even without a known cause for hyperuricemia, are at increased risk of developing gouty arthritis. This presentation suggests that psoriasis can be an independent risk factor for gouty attacks, though this requires further larger studies.
\end{abstract}

Keywords: gout, hyperuricemia, psoriasis.

\section{Introduction}

Psoriasis is a chronic inflammatory disease typically involving skin, joints, nails, and scalp [1]. Several reports have described the association of psoriasis with metabolic derangements such as metabolic syndrome and hyperuricemia [2, 3]. Psoriatic arthritis ((PsA) is an inflammatory seronegative arthritis which can occur in up to one-fourth of patients with psoriasis [4]. There are case reports in the literature describing the simultaneous occurrence of psoriasis and gout [1, 5-7]. These reports have revealed that the risk of gout is increased with psoriasis, particularly among patients with PsA [4]. However, there are conflicting reports regarding this finding. For instance, in a populationbased study, it was concluded that psoriasis per se is not a risk factor for gout or hyperuricemia [8].

An association of gout with extensive long-standing psoriasis may exist, particularly in male patients who have additional risk factors for hyperuricemia. Evidence is growing regarding the nature of association between psoriasis and gout. Herein is presented the case of simultaneous psoriasis and gouty arthritis. It is suggested that reports like this one will better elucidate the nature of the association between these two conditions.

\section{Case report}

The patient was a 53-year-old male who referred to the rheumatology department of a tertiary hospital because of an acute gouty attack in his knees and feet bilaterally. He was a smoker and had a 30-year history of extensive psoriasis. Review of the patient's medical records revealed that the diagnosis of psoriasis had been made when he was 23 years old. At that time, a skin biopsy had been done for his white squamous lesions, and the diagnosis of psoriasis had been confirmed on histopathologic examination. His family history was positive for psoriasis in his father.

The current gouty attack had started two days earlier. Upon presentation, the patient was in no acute distress and his vital signs were normal. His weight was $62 \mathrm{~kg}$ and his height was $158 \mathrm{~cm}$ (BMI= 24.9 $\mathrm{kg} / \mathrm{m}^{2}$ ). On examination, swollen and tender knee, first metatarsophalangeal, and subtalar joints were detected

* Corresponding Authors: Zeinab Saremi, Email: z13612002@yahoo.com; Tel: +98 5138012753

Received: 14 May 2017; Accepted: 05 July 2017 
bilaterally. The patient did not have "sausage digits". Nail pitting was observed in some of his toenails. In addition, widespread guttate psoriasis affecting the trunk, legs, and arms as well as scalp plaques were noted (Figs. 1 and 2). The patient also had typical terminal interphalangeal involvement of psoriatic arthritis with tenosynovitis (Fig. 3). He had experienced worsening of his skin condition recently, and topical corticosteroid had been administered accordingly. In addition, he was taking methotrexate (15 mg weekly) and folic acid for his psoriasis.

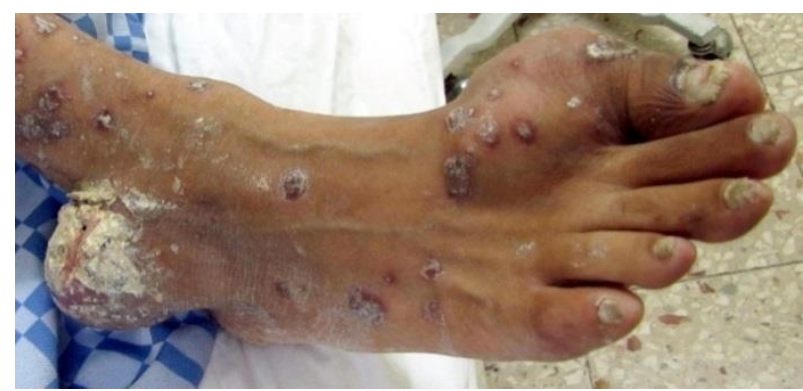

Fig. 1. Tender and swollen first metatarsophalangeal and subtalar joints diagnosed as tophaceous gout in a patient with long standing psoriasis

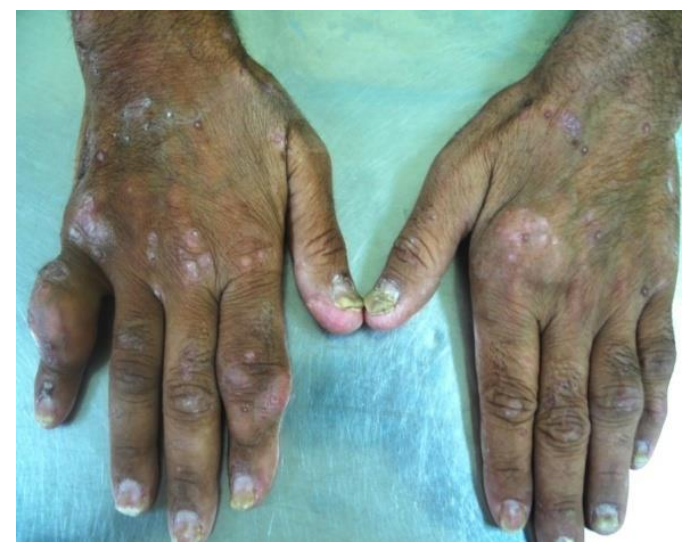

Fig. 2. Psoriatic lesions on the hands of a patient who developed gout

The patient had been visited two years earlier following a severe attack of pain and swelling in his right first metatarsophalangeal joint. He stated that he did not seek medical attention and did not receive any medication for his first gouty attack.

Knee joint aspiration was performed and showed 810 white blood cells/hpf (89\% polymorphonuclear cells) and numerous monosodium urate monohydrate (MSUM) crystals. Routine laboratory test results showed a normal CBC, elevated serum uric acid level (10 mg/dL), elevated ESR (79 mg/dL), and CRP (59 $\mathrm{mg} / \mathrm{L}$ ), normal creatinine, decreased vitamin $\mathrm{D}$, and normal calcium, phosphorus, and magnesium. Kidney ultrasound was unremarkable. The 24-hour urinary uric acid level was $1,100 \mathrm{mg}$ (upper normal limit= 750 $\mathrm{mg}$ /day). The patient's other laboratory examinations showed a triglyceride level of $160 \mathrm{mg} / \mathrm{dL}$, total cholesterol of $136 \mathrm{mg} / \mathrm{dL}$, HDL of $40 \mathrm{mg} / \mathrm{dL}$, and a fasting blood sugar (FBS) of $109 \mathrm{mg} / \mathrm{dL}$. The rheumatoid factor was negative. These findings suggested the diagnosis of concurrent psoriasis and gout.

Considering the involvement of several joints, oral prednisolone (60 mg daily) was initiated for 3 days; after improvement in the inflammation was observed, prednisolone $30 \mathrm{mg}$ daily was continued for two weeks and then tapered off over a 4-week period to $5 \mathrm{~g}$ daily. Treatment resulted in a regression of pain and effusion over one week.

Colchicine (1 mg daily), allopurinol (300 mg daily), and sulfasalazine ( $2 \mathrm{~g}$ daily) were added to the patient's medications and a follow-up was initiated. The patient had no further joint complaints when seen at his 6-month follow-up. Following discharge, he has remained asymptomatic but has a persistently elevated serum uric acid.

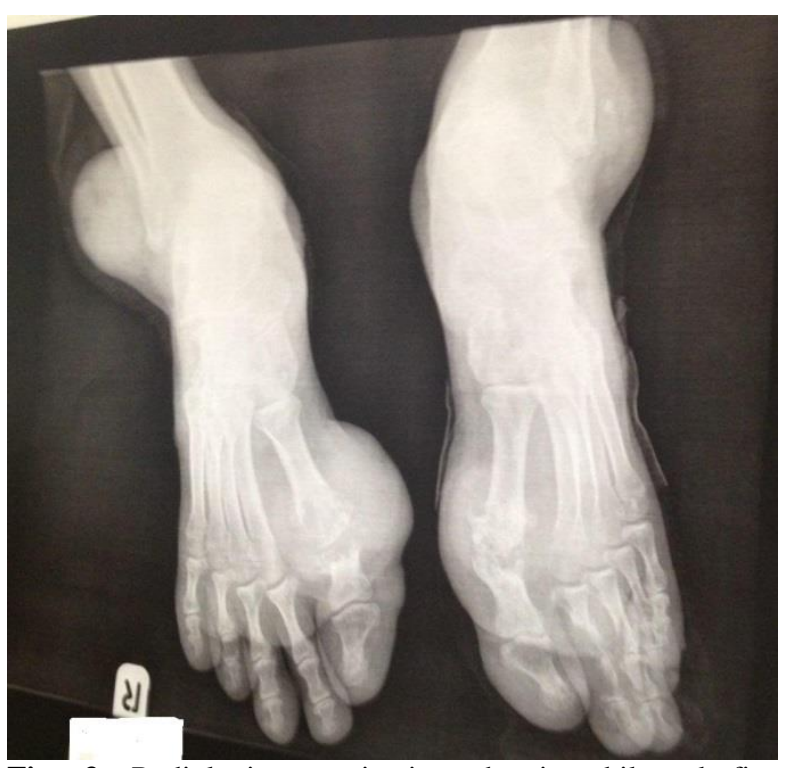

Fig. 3. Radiologic examination showing bilateral first metatarsophalangeal and subtalar joint erosions

\section{Discussion}

Herein is presented the case of a patient with longstanding psoriasis who developed gouty attack in several joints. Gout is an inflammatory crystal arthropathy characterized by deposition of monosodium urate crystals in the joints. Hyperuricemia 
is a major factor in the occurrence of gout [4]. This association has been reported previously. Lobato et al. [1] reported a 47-year-old man with a long history of psoriasis who presented with worsening of skin lesions as well as a swollen and painful left foot. Similar to the patient in the current report whose joint involvement developed gradually, the patient reported by Lobato et al. also developed his joint symptoms over a 6-month period. The described patient [1] also had hyperuricemia. With the administration of colchicine and allopurinol, his symptoms resolved and his serum uric acid level normalized.

There is evidence that hyperuricemia can develop in psoriasis and this per se may result in the development of gout. The patient presented herein had normal kidney function and was not taking any medication that could result in hyperuricemia. It is believed that hyperuricemia is the result of increased cell turnover in psoriasis [1]. In the presented patient, increased inflammatory markers including ESR and CRP were observed. This finding has also been reported earlier and reflects increased cell turnover and overall inflammation which are associated with psoriasis severity [9]. The presented patient also had worsening of his skin lesions with concomitant gouty attack. The patient described by another case report also showed concomitant worsening of skin lesions and the presence of gout [1]. There is concern among dermatologists that administering systemic corticosteroids in psoriasis can deteriorate psoriatic lesions [10]. As several joints were involved in the presented patient, systemic corticosteroid was started. Worsening of psoriatic lesions did not occur. In a review article regarding systemic steroids in the treatment of psoriasis, the authors concluded that a reevaluation of this issue is necessary [11]. Metabolic syndrome is another consideration that has been reported to occur more frequently in psoriasis patients who develop hyperuricemia, although it is premature to suggest a causal relationship, and more studies are required [2]. In a systematic review on more than 40,000 patients with psoriasis, it was found that not only is metabolic syndrome more prevalent when compared to the general population, but the severity of psoriasis is also significantly correlated with a higher likelihood of developing metabolic syndrome [12]. The presented patient did not meet the criteria of metabolic syndrome, although his BMI was in the upper normal range. In several cross-sectional studies, psoriasis was recognized by regression methods as an independent factor for hyperuricemia when adjustments were made for age, gender, and metabolic syndrome [2]. Whether metabolic syndrome in psoriatic patients can contribute directly to gouty attacks seems unclear with the current evidence.

The medications administered to the presented patient were the same conventional, established treatments used in gouty attacks in patients without psoriasis. The treatments resulted in an improvement of symptoms, and on further follow-up no gouty attack recurrence was observed.

\section{Conclusion}

Patients with psoriasis are at increased risk for hyperuricemia which can result in the development of gouty arthritis. The presented patient did not have any other risk factors for hyperuricemia or gout. Hence, is it suggested that psoriasis is an independent risk factor for hyperuricemia or gout. This report provides the basis for further studies with two objectives. First, it is of great importance to know which psoriatic patients are at increased risk for hyperuricemia and gout; secondly, it is advantageous to determine whether the administration of preventive medications could be helpful.

\section{Conflicts of interest}

The author declares no conflicts of interest.

\section{Acknowledgments}

The authors thank the patient who allowed us to report this case and the personnel of the Rheumatology Clinic, Gheam Hospital, Mashhad University of Medical Sciences, Mashhad, Iran.

\section{References}

1. Lobato LC, Coutinho JC, Frota MZ, Schettini AP, Santos M. Chronic tophaceous gout in patients with psoriasis. An Bras Dermatol 2017; 92(1): 104-106. doi: $\quad 10.1590 /$ abd1806-4841. 20174895

2. Gisondi P, Targher G, Cagalli A, Girolomoni G. Hyperuricemia in patients with chronic plaque psoriasis. J Am Acad Dermatol 2014; 70(1): 127-30. doi: 10.1016/j.jaad.2013.09.005.

3. Gisondi P, Targher G, Zoppini G, Girolomoni G. Non-alcoholic fatty liver disease in patients with chronic plaque psoriasis. $J$ Hepatol 2009; 51(4): 758-64. doi: 10.1016/j.jhep.2009.04.020.

4. Merola JF, Wu S, Han J, Choi HK, Qureshi AA. Psoriasis, psoriatic arthritis and risk of gout in US men and women. Ann Rheum Dis 2015; 74(8): 1495500. doi: 10.1136/annrheumdis2014-205212.

5. Turan Y, Kocaağa Z, Koçyiğit H,

Rheum. Res., Vol. 3, No. 1, Jan. 2017 

Gürgan A, Korhan B.
Coexistence Spondyloarthropathy and Gout: A Case Report. Turkish Journal of Rheumatology 2009; 24(3).

6. Restrepo Escobar M, González Naranjo LA, Vásquez Duque GM. Coexistence of psoriatic spondylitis and gout: a case report. Revista Colombiana de Reumatología 2011; 18(3): 247250. doi: 10.1016/s0121-8123(11) 70057-3.

7. Liu M, Li JH, Li B, He CD, Xiao T, Chen HD. Coexisting gout, erythrodermic psoriasis and psoriatic arthritis. Eur J Dermatol 2009; 19(2): 184-5.
8. Lai YC, Yew YW. Psoriasis and uric acid: a population-based cross-sectional study. Clin Exp Dermatol 2016; 41(3): 260-6. doi: 10.1111/ced.12781.

9. Kwon HH, Kwon IH, Choi JW, Youn JI. Cross-sectional study on the correlation of serum uric acid with disease severity in Korean patients with psoriasis. Clin Exp Dermatol 2011; 36(5): 473-8. doi: 10.1111/j.1365-2230.2010.03988. $\mathrm{x}$.

10. Brenner M, Molin S, Ruebsam K, Weisenseel P, Ruzicka T, Prinz JC. Generalized pustular psoriasis induced by systemic glucocorticosteroids: four cases and recommendations for treatment. Br J Dermatol 2009; 161(4): 964-6. doi: 10.1111/j. 1365-2133.2009.09348.x.

11. Mrowietz U, Domm S. Systemic steroids in the treatment of psoriasis: what is fact, what is fiction? J Eur Acad Dermatol Venereol 2012; 27(8): 1022-5. doi: $\quad 10.1111 / \mathrm{j} .1468-3083.2012$. 04656.x.

12. Armstrong AW, Harskamp CT, Armstrong EJ. Psoriasis and metabolic syndrome: a systematic review and meta-analysis of observational studies. J Am Acad Dermatol 2013; 68(4): 654-62. doi: 10.1016/j.jaad.2012.08.015. 